\title{
Fatty Acid Composition of Symbiotic Cyanobacteria from Different Host Plant (Azolla) Species: Evidence for Coevolution of Host and Symbiont
}

\author{
RODULIO CAUDALES, ${ }^{1 *}$ JOHN M. WELLS, ${ }^{2}$ ALAN D. ANTOINE, ${ }^{1}$ AND JAMES E. BUTTERFIELD ${ }^{2}$ \\ Department of Biochemistry and Microbiology, Rutgers-The State University of New Jersey, New Brunswick, \\ New Jersey 08903-0231, ${ }^{1}$ and Agricultural Research Service, U.S. Department \\ of Agriculture, Philadelphia, Pennsylvania $19118^{2}$
}

\begin{abstract}
The total cellular fatty acid contents of $\mathbf{4 0}$ recently isolated cyanobacterial symbionts obtained from seven species of Azolla host plants were determined by gas-liquid chromatography-mass spectroscopy. A total of 63 fatty acids belonging to seven distinct chemical classes were identified. Fatty acid compositions varied among the cyanobacteria depending on the hosts species. Parameters that differed significantly (at the $99 \%$ level of probability) included the concentrations of the $16: 0$ and 18:3 fatty acids, the total concentrations of the polyunsaturated acids, the total concentrations of the 16-carbon and 18-carbon fatty acids, the ratios of unsaturated fatty acids to saturated fatty acids, and the total percentages of straight-chain even-carbonnumber fatty acids, unsaturated fatty acids, and branched-chain unsaturated fatty acids. The results of an analysis of variance suggested statistical regression for the total percentages of these fatty acids and chemical classes according to the following linear alignment of cyanobacteria by host: Azolla filiculoides, Azolla microphylla, Azolla caroliniana, Azolla mexicana, Azolla rubra, Azolla nilotica, and Azolla pinnata (including Azolla pinnata subsp. pinnata and Azolla pinnata subsp. imbricata). The seven groups could be divided into two distinct clusters on the basis of the results of a dendrogram analysis of Euclidian distances. The symbionts obtained from $A$. filiculoides, $A$. microphylla, $A$. mexicana, and $A$. caroliniana constituted one cluster, and the symbionts obtained from $A$. rubra, $A$. nilotica, and $A$. pinnata constituted a second cluster. A minor dichotomy separated the $A$. filiculoides symbionts from the other members of the first cluster. The clustering of $A z$ olla cyanobacterial symbionts based on the results of our fatty acid analysis correlates remarkably well with the taxonomic grouping of the American Azolla species. This correlation suggests that the cyanobacterial symbionts of $A z$ olla spp. coevolved into distinct genetic groups with their hosts.
\end{abstract}

The cyanobacteria are a diverse group of oxygenic, photosynthetic prokaryotes which occur worldwide in marine, aquatic, and terrestrial habitats (8). Many genera of cyanobacteria are symbiotically associated with plants, fungi (lichens), animals, and bacteria. The genera Anabaena and Nostoc are the major genera whose members are found in plants (23).

Members of the water fern genus Azolla are hosts of cyanobacterial symbionts that occur in specially adapted cavities in the upper leaf fronds $(7,16,18)$. There are seven $A z o l l a$ species belonging to two subgenera, each of which has a distinct morphology; Azolla caroliniana, Azolla filiculoides, Azolla mexicana, Azolla microphylla, and Azolla rubra are members of the subgenus Azolla, and Azolla nilotica and Azolla pinnata are members of the subgenus Rhizosperma. A. pinnata contains two subspecies, $A$. pinnata subsp. pinnata and $A$. pinnata subsp. imbricata $(13,17)$. Using plant morphology, ecophysiology, isozyme, and DNA fingerprint criteria, Zimmerman et al. (2527 ) found that $A$. caroliniana, $A$. mexicana, and $A$. microphylla are closely related phylogenetically and that $A$. filiculoides and A. rubra are not as closely related.

The Azolla symbionts are morphologically diverse, particularly in their cellular dimensions $(1,11)$ and the arrangement of their vegetative and reproductive cells in filaments (1). The cyanobacteria whose hosts are members of the two subgenera (subgenera Azolla and Rhizosperma, the latter including $A$.

\footnotetext{
* Corresponding author. Present address: Department of Biological Sciences, Botswana University, Private Bag 0022, Gaborone, Botswana. Phone: 267-351151, ext. 2604. Fax: 267-356591. Electronic mail address: caudales@motswedi.ub.bw.
}

rubra) have characteristic morphological differences related to the growth and development of their reproductive cells, such as hormogonia and akinetes $(1,2)$. In addition, analyses of DNA restriction fragment length polymorphisms in cyanobacterial DNAs have revealed differences between symbionts whose hosts are members of the two subgenera at the molecular level $(9,10,15,21,22)$.

Fatty acid composition is a useful analytical tool in bacterial taxonomy, and the fatty acid compositions of a limited number of Azolla cyanobacterial symbionts have been determined previously; wide ranges of values have been obtained for some important components in these analyses $(3,6)$. It is not clear if the variability observed was due to strain differences or was related to the host species. In this study we examined the variations in the fatty acid compositions of 40 different strains of cyanobacteria isolated from the seven Azolla species and in particular tried to detect differences related to the hosts of the organisms.

\section{MATERIALS AND METHODS}

Media and growth conditions. Azolla plants were grown in defined mineral medium without nitrogen (3). The photon flux density was $100 \mu \mathrm{mol} \mathrm{m} \mathrm{m}^{-2} \mathrm{~s}^{-1}$ and was provided by a combination of cool white fluorescent and incandescent light; a 14-h light-10-h dark cycle was used (19). The temperature was $23 \pm 1{ }^{\circ} \mathrm{C}$ during the light periods and $17 \pm 1^{\circ} \mathrm{C}$ during the dark periods. The cyanobacterial strains which we used and their geographic origins and sources are listed in Table 1.

Cyanobacterial symbionts were harvested from 14- to 18-day-old plants as previously described (3) and were stored at $-20^{\circ} \mathrm{C}$ for subsequent fatty acid analysis.

Fatty acid analysis. Preparations of cyanobacterial cells were saponified, esterified, and then analyzed to determine their fatty acid contents by gas-liquid chromatography-mass spectroscopy, as previously described, using a Varian 
TABLE 1. Origins of Azolla host plants used in this study and designations of symbiotic cyanobacterial strains

\begin{tabular}{|c|c|c|c|c|}
\hline Host $^{a}$ & Bacterial strain & $\begin{array}{c}\text { Original } \\
\text { designation }\end{array}$ & Geographic origin & Source $^{b}$ \\
\hline \multirow[t]{5}{*}{ A. caroliniana } & $\mathrm{C} 1$ & $\mathrm{C} 1$ & Virginia & G.P. \\
\hline & $\mathrm{C} 2$ & 3010 & Rio Salimões, Ilha Paciência, Amazonas, Brazil & W.Z. \\
\hline & $\mathrm{C} 3$ & 3014 & Rio Negro, Catalão, Amazonas, Brazil & W.Z. \\
\hline & $\mathrm{C} 4$ & & La Esperanza, Manatí, Puerto Rico & R.C. \\
\hline & $\mathrm{C} 8$ & & Río Chavón, Dominican Republic & R.C. \\
\hline \multirow[t]{9}{*}{ A. filiculoides } & $\mathrm{F} 1$ & $81 \mathrm{FI}$ & Lima, Peru & V.H. \\
\hline & F8 & 6005 & Kyoto Tanabe-Cho, Japan & W.Z. \\
\hline & F10 & $103 \mathrm{FI}$ & Hidalgo, Mexico & V.H. \\
\hline & F12 & $126 \mathrm{FI}$ & Karoo, South Africa & V.H. \\
\hline & F13 & $159 \mathrm{FI}$ & Victorville, Calif. & V.H. \\
\hline & F14 & $162 \mathrm{FI}$ & Tristan da Cunha, United Kingdom & V.H. \\
\hline & F15 & 167FI & Pondolivado, Greece & V.H. \\
\hline & F18 & $185 \mathrm{FI}$ & Montevideo, Uruguay & V.H. \\
\hline & C9 & $208 \mathrm{CA}$ & Fyrish, Guyana & V.H. \\
\hline \multirow[t]{5}{*}{ A. mexicana } & $\mathrm{X} 1$ (= BR-S-CF) & $\mathrm{X} 1$ & California & G.P. \\
\hline & $\mathrm{X} 4$ & & Mexico City, Mexico & R.O. \\
\hline & $\mathrm{X} 5$ & 54 & Guyana & W.R. \\
\hline & $\mathrm{X} 8$ & 58 & California & W.R. \\
\hline & $\mathrm{X} 10$ & & Illinois & R.C. \\
\hline \multirow[t]{4}{*}{ A. microphylla } & M1 & $65 \mathrm{MI}$ & Paraíso do Norte, Brazil & V.H. \\
\hline & M2 (= TL-GI-CF) & M2 & Galápagos, Ecuador & G.P. \\
\hline & M4 & $104 \mathrm{MI}$ & Sinaloa, Mexico & V.H. \\
\hline & M5 & 4032 & & w.Z. \\
\hline \multirow[t]{5}{*}{ A. rubra } & RF3 (= RC-B1-CF) & F3 & Hawaii & G.P. \\
\hline & R1 & 163RU & Victoria, Australia & V.H. \\
\hline & R2 & $002 R U$ & North Island, New Zealand & V.H. \\
\hline & R3 & 204RU & Nouville, New Zealand & V.H. \\
\hline & R4 & 200RU & South Island, New Zealand & V.H. \\
\hline \multirow[t]{7}{*}{ A. nilotica } & N1 & $82 \mathrm{NI}$ & Kosti, Sudan & V.H. \\
\hline & & N1 & Kosti, Sudan & G.P. \\
\hline & & 5001 & Kosti, Sudan & W.Z. \\
\hline & N1-SC & $82 \mathrm{NI}$ & Kosti, Sudan & V.H. \\
\hline & & N1 & Kosti, Sudan & G.P. \\
\hline & & 5001 & Kosti, Sudan & W.Z. \\
\hline & $\mathrm{N} 2$ & $15 \mathrm{NI}$ & Burundi & V.H. \\
\hline \multirow[t]{5}{*}{ A. pinnata subsp. imbricata } & I1 (= K2B-T-CF $)$ & I1 & Shoolong, People's Republic of China & G.P. \\
\hline & I2 & TDP15 & Bankok, Thailand & W.Z. \\
\hline & I3 & JNPI1 & Chisato Mie, Japan & W.Z. \\
\hline & I4 & 73 & Indonesia & W.Z. \\
\hline & I5 & $36 \mathrm{PI}$ & Senegal & W.Z. \\
\hline \multirow[t]{3}{*}{ A. pinnata subsp. pinnata } & P1 (= BR-M-CF) & P1 & Malaysia & G.P. \\
\hline & P1-SC (= BR-M-CF) & P1 & Malaysia & G.P. \\
\hline & P4 & $136 \mathrm{PP}$ & Sierra Leone & V.H. \\
\hline
\end{tabular}

${ }^{a} A$. caroliniana, A. filiculoides, $A$. mexicana, $A$. microphylla, and $A$. rubra are members of the subgenus $A z o l l a$, and $A$. nilotica and $A$. pinnata are members of the subgenus Rhizosperma.

${ }^{b}$ G.P., Gerald Peters, Virginia Commonwealth University, Richmond; R.C., Rodulio Caudales, Rutgers-The State University of New Jersey, New Brunswick; R.O., Roxana Olvera, Mexico City, Mexico; W.Z., William Zimmerman, University of Michigan, Dearborn; V.H., Charles Van Hove, Université Catholique de Louvain, Louvain-la-Neuve, Belgium; W.R., William Rains, University of California, Davis.

model 3700 gas chromatography equipped with a flame ionization detector and a capillary glass column ( $15 \mathrm{~m}$ by $0.25 \mathrm{~mm}$ ) coated with SPB-1 (Supelco, Inc., State College, Pa.) as the nonpolar stationary phase $(3,5)$. Peaks were identified by cochromatography with authentic standards (Supelco, Inc.), by chemical confirmatory tests, and by mass spectroscopy, as described previously (5). Each cyanobacterial sample was analyzed once, and the average for each fatty acid component was determined by using the data for all isolates obtained from each host species. Cyanobacteria obtained from $A$. nilotica (strain N1), A. rubra (strain $\mathrm{R} 1$ ), and $A$. pinnata subsp. pinnata (strain $\mathrm{P} 1$ ) were analyzed seven, five, and three times, respectively, in order to determine the level of variability due to the experimental procedure used.

Statistical analysis. Statistical differences between values obtained for selected fatty acids and between ratios were determined by performing an analysis of variance, using Duncan's multiple-range test (24). Differences were considered significant at the $95 \%$ level of probability $(P \geq 0.05)$. The fatty acid data for cyanobacterial isolates obtained from the two subspecies of $A$. pinnata were averaged in order to increase the total sample number for the group and because there were no statistically significant differences between the compositions of the two populations.

The variability in the values from repeated fatty acid analyses performed with the same strain (i.e., variability due to experimental procedure) was determined by calculating proximity coefficients. These values were derived by constructing a model profile for 40 strains of cyanobacteria on the basis of the average values shown in Table 2 and calculating the aggregate levels of difference for the individual fatty acids obtained from each sample used for the model.

A cluster analysis of the Euclidian distances between taxonomic groups was performed and a dendrogram was constructed by using the STATPRO statistical software (Wadsworth Professional Software, Inc., Boston, Mass.). We selected fatty acid components that exhibited statistically significant differences as a function of host species. The values which we obtained were then used as input data for a statistical matrix in the cluster analysis, from which the dendrogram was constructed. The dendrogram was based on group distances obtained from a pair-weighted cluster analysis.

\section{RESULTS}

General features of fatty acid profiles. We identified 63 different fatty acids, and these fatty acids were present at levels that were consistent with the levels described in previously 


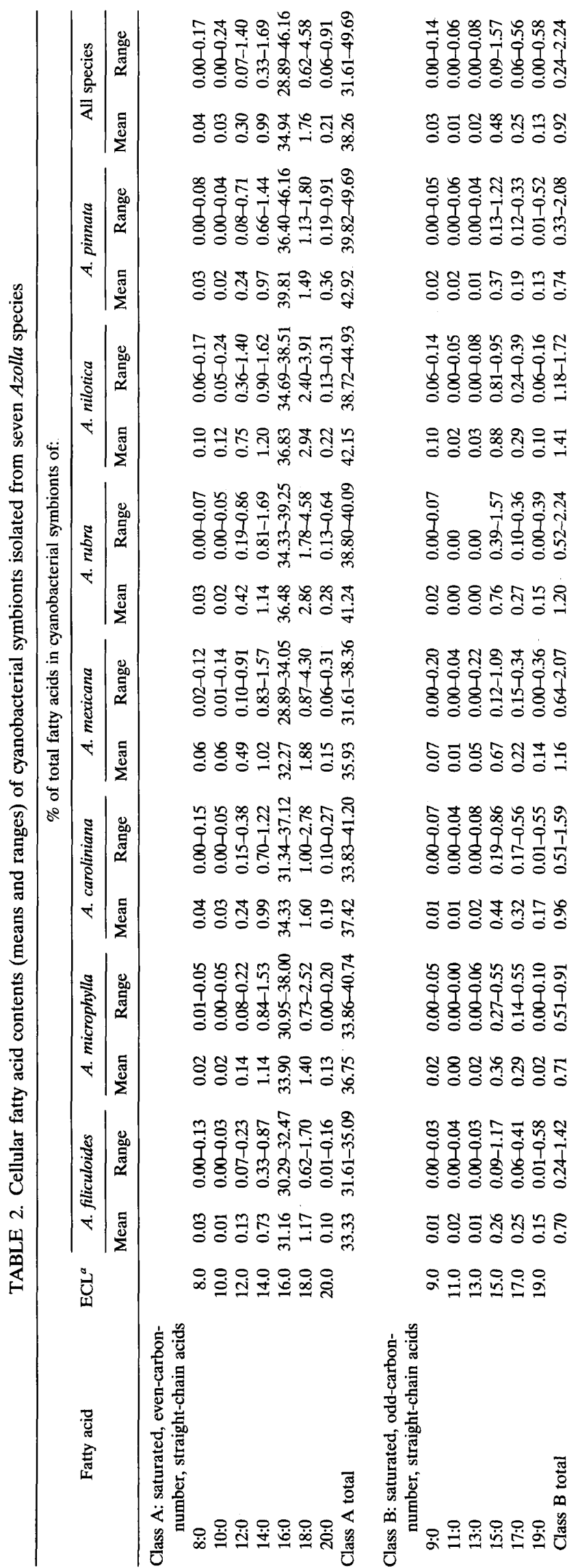

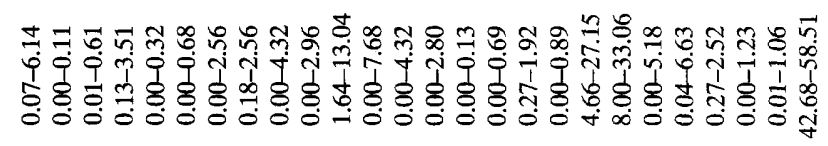

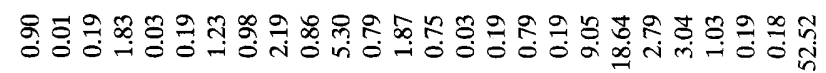

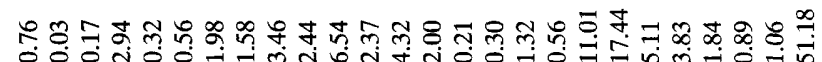

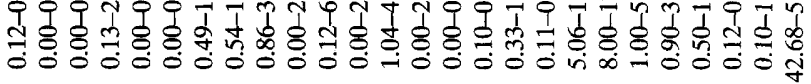

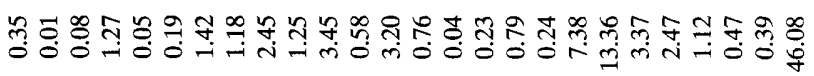

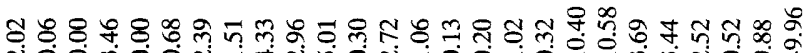

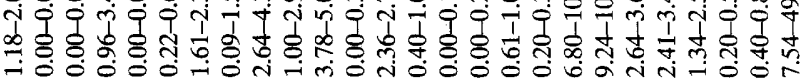

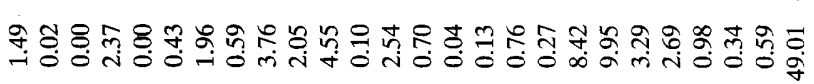

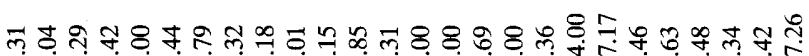
फ.

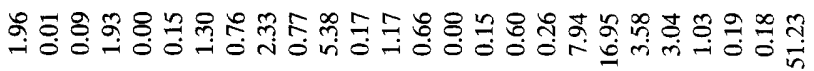

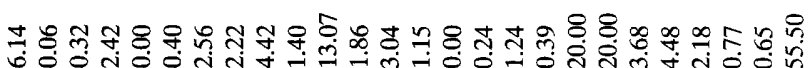

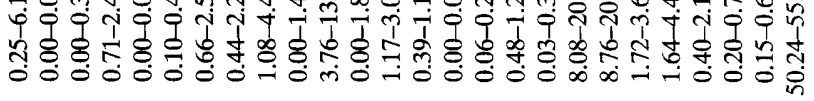

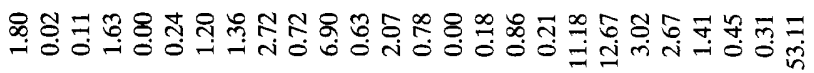

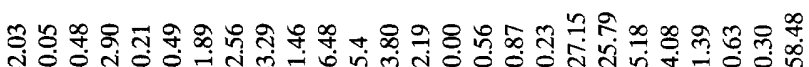

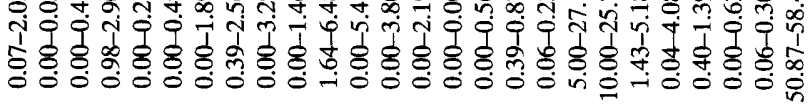

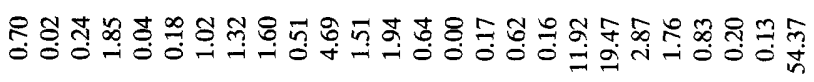

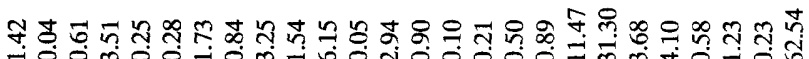

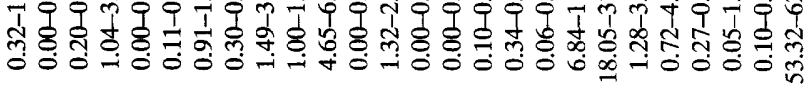

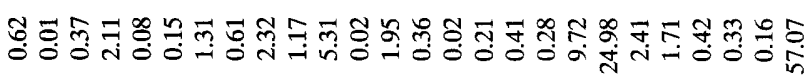

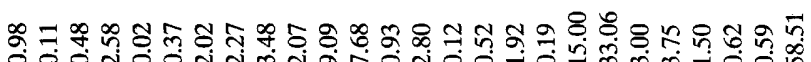

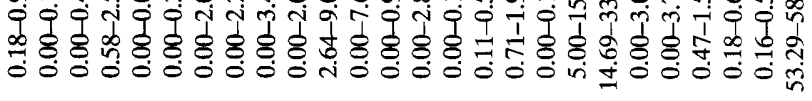

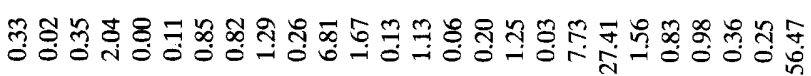

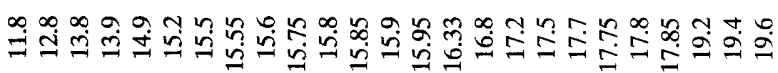

然

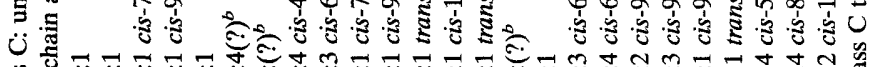

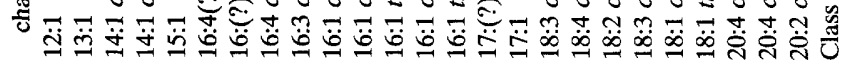




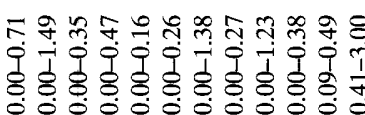

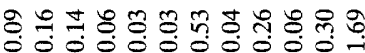

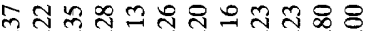

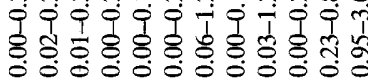

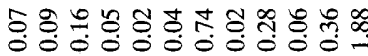

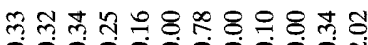

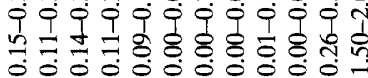

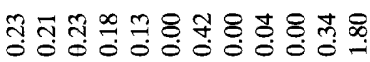

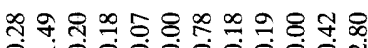

1.

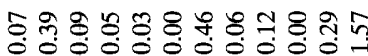

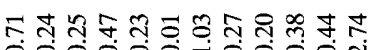

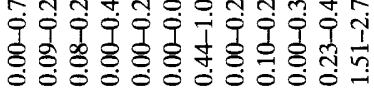

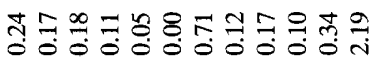

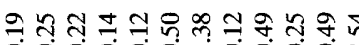

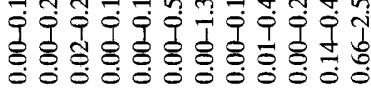

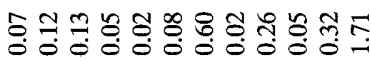

ธำ 888 을 ㅊำ ำ

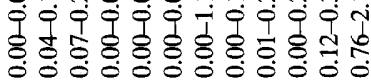

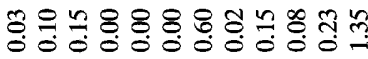

우으ำำ

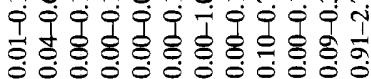

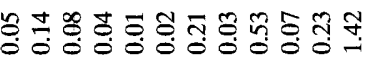

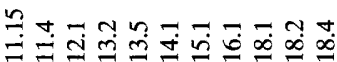

:

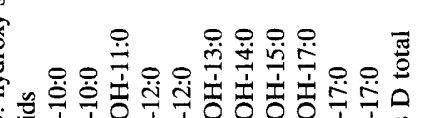

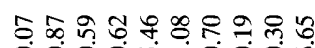

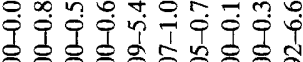

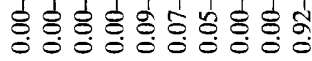

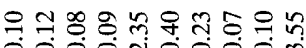

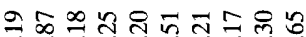

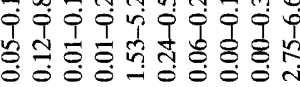

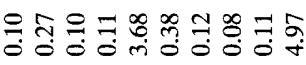

는ำกำำ

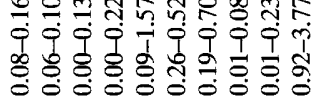

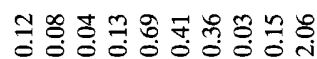

눈ำ

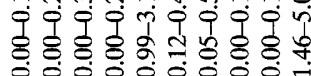

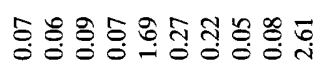

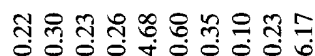

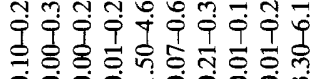

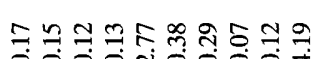

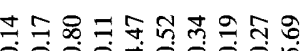

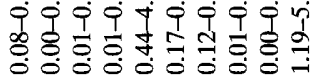

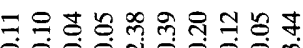

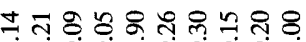

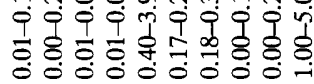

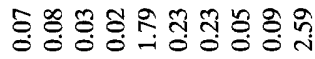

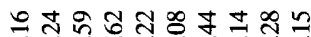

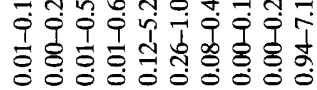

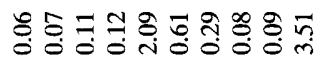

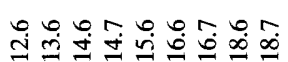

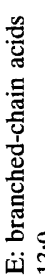

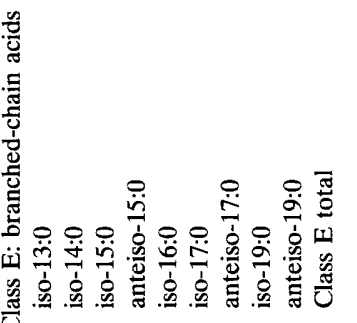

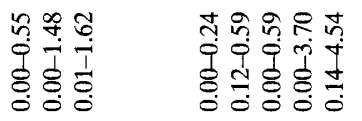

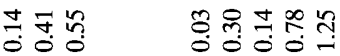

ले 古 भิ

2 ำกำ

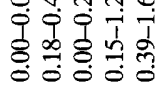

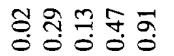

ㄱํㅇํํㅇ

กำ

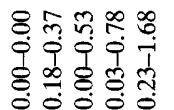

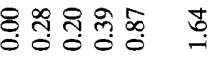

ขึํำ

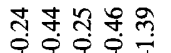

옹용영

m.

꼭

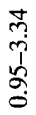

$\stackrel{\infty}{-}$

in

क्ष

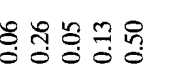

영ํํㅇ

సิ ติ

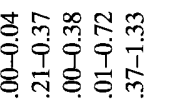

ㅇำ

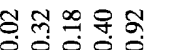

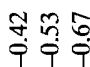

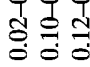

țํㅇํㅇ

0
0
0
0
0
0
0
0
0

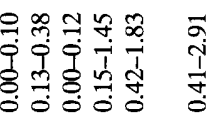

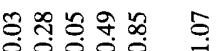

ะสำ

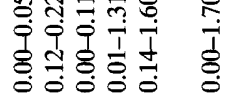

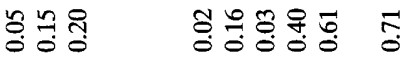

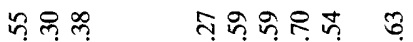

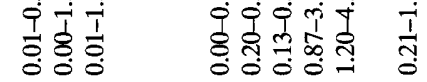

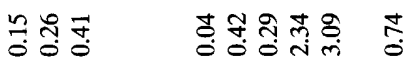

ดे

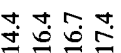

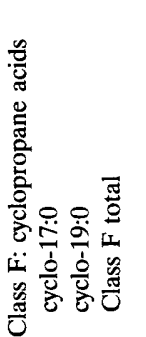

㝕

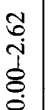

ঙำ

$\stackrel{\circ}{-1}$

i.

可

i



吉

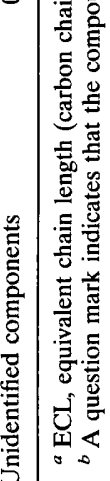


TABLE 3. Variability in fatty acid analysis data obtained after repeated extractions of cyanobacteria from three different Azolla hosts

\begin{tabular}{lccc}
\hline \multicolumn{1}{c}{ Host $^{a}$} & $\begin{array}{c}\text { Symbiont } \\
\text { strain }\end{array}$ & $\begin{array}{c}\text { No. of } \\
\text { replicates } \\
\text { examined }^{b}\end{array}$ & $\begin{array}{c}\text { Avg\% } \\
\text { Proximity to }_{\text {group model }^{c}}\end{array}$ \\
\hline $\begin{array}{l}\text { A. nilotica } \\
\text { A. rubra }\end{array}$ & $\mathrm{N} 1$ & 7 & $80.1 \pm 4.07$ \\
A. pinnata subsp. pinnata & $\mathrm{R} 1$ & 5 & $70.8 \pm 2.92$ \\
\hline
\end{tabular}

${ }^{a}$ Plants were grown in defined mineral medium without nitrogen as described in the text.

${ }^{b}$ Cyanobacteria were extracted from 14- to 18-day-old plants harvested at different times.

$c$ Average \pm standard deviation proximity values for individual fatty acid profiles compared with a group model composed of the profiles of 40 different strains. None of the values was statistically significantly different.

published reports, in which smaller numbers of cyanobacterial samples were analyzed. Sixteen major fatty acid components (each constituting more than $0.9 \%$ of the total fatty acids) accounted for $87.92 \%$ of the total fatty acids. These major components included the saturated 14:0 $(0.99 \%), 16: 0(34.94 \%)$, and 18:0 $(1.76 \%)$ fatty acids; the unsaturated straight-chain $12: 1(0.90 \%), 14: 1$ cis-9 (1.83\%), 16:1 cis-9 (5.30\%), 16:1 cis-11 (1.87\%), 16:3 cis-6 (2.19\%), 16:4 cis-4 (0.98\%), 18:2 cis-9 (9.05\%), 18:3 cis-9 (18.64\%), 18:1 cis-9 (2.79\%), 18:1 trans-9 $(3.04 \%)$, and $20: 4$ cis-5 $(1.03 \%)$ fatty acids; and the branchedchain iso- $16: 0$ fatty acid $(2.35 \%)$ (Table 2$)$.

We detected and identified 44 minor fatty acid components which accounted for $12.04 \%$ of the total fatty acids and three components that were present at trace levels. These included hydroxy-substituted fatty acids $(1.69 \%)$, branched-chain fatty acids other than the iso-16:0 fatty acid (which accounted for $2.35 \%$ of the total of $3.55 \%$ determined for this class), and cyclopropane acids $(0.55 \%)$. We also detected an incompletely characterized unsaturated 16 -carbon fatty acid $(1.23 \%)$ with an equivalent chain length of 15.5 . The most abundant fatty acid was the 16:0 fatty acid, which accounted for between 28.89 and $46.16 \%$ of the total fatty acids (Table 2).

Variability due to experimental procedures. The differences in the fatty acid compositions of individual strains due to the experimental procedures which we used were not significant statistically. The average proximity coefficient obtained when we compared the results of seven replicate analyses of cya- nobacterial isolate $\mathrm{N} 1$ obtained from $A$. nilotica with the results obtained for the entire $A$. nilotica group (Table 2) was $80.1 \%$, and the individual proximity coefficients ranged from 76.2 to $83.3 \%$ (all with a standard deviation of $\pm 4.07 \%$ ). Similarly, the differences in the data for the two other strains analyzed were not statistically significant (Table 3 ).

Variability due to host. We observed statistically significant differences in the fatty acid compositions of cyanobacteria obtained from different hosts. Differences in the following parameters were significant at a level of $99 \%$ : concentrations of the $16: 0$ and $18: 3$ fatty acids, total concentrations of the polyunsaturated fatty acids, 16-carbon and 18-carbon fatty acids, class A fatty acids (straight-chain saturated fatty acids), class $C$ fatty acids (straight-chain unsaturated fatty acids), and class $G$ fatty acids (branched-chain unsaturated fatty acids), and ratios of unsaturated fatty acids (classes $C$ and $G$ ) to saturated fatty acids (classes $A$ and $B$ ) (Table 4 ).

The results of an analysis of variance suggested that the cyanobacterial symbionts obtained from the seven hosts could be positioned along a continuum on the basis of the values obtained for the variable fatty acid parameters. The cyanobacterial symbionts obtained from $A$. filiculoides were at one end of the continuum, the symbionts obtained from $A$. caroliniana, $A$. mexicana, $A$. microphylla, and $A$. rubra were in intermediate positions, and the symbionts obtained from $A$. nilotica, $A$. pinnata subsp. pinnata, and $A$. pinnata subsp. imbricata (the latter two as a combined group) were at the other end. Segregation of the host species into clusters along the continuum was evident when the data were subjected to an analysis of Euclidian distances and a dendrogram was constructed.

The dendrogram which we constructed indicated that the cyanobacteria obtained from the seven Azolla host species segregated into two main clusters, with a secondary dichotomy in one of these clusters (Fig. 1). The symbionts obtained from $A$. pinnata, $A$. nilotica, and $A$. rubra constituted the first cluster, and the other Azolla symbiont cluster was composed of symbionts obtained from $A$. filiculoides, $A$. microphylla, $A$. mexicana, and $A$. caroliniana. The symbionts obtained from $A$. filiculoides were separated from the other symbionts by a secondary dichotomy.

\section{DISCUSSION}

Host species have been shown to be significant sources of differences in the fatty acid compositions of populations of

TABLE 4. Alignment of cyanobacterial symbionts obtained from seven Azolla species based on statistical differences in fatty acid parameters

\begin{tabular}{|c|c|c|c|c|c|c|c|c|c|c|c|}
\hline \multirow{3}{*}{ Host species } & \multicolumn{9}{|c|}{$\%$ of the following fatty acids in cyanobacterial symbionts ${ }^{a}$ : } & \multicolumn{2}{|c|}{ Ratios $^{a}$} \\
\hline & \multicolumn{2}{|c|}{ Class A fatty acids } & \multicolumn{3}{|c|}{ Class $C$ fatty acids } & \multirow{2}{*}{$\begin{array}{l}\text { Class } \\
\text { G fatty } \\
\text { acids }\end{array}$} & \multirow[b]{2}{*}{$\begin{array}{c}\text { All } \\
\text { PUFA }\end{array}$} & \multirow[b]{2}{*}{$\begin{array}{c}\text { All } C_{16} \\
\text { fatty acids }\end{array}$} & \multirow[b]{2}{*}{$\begin{array}{c}\text { All } C_{18} \\
\text { fatty acids }\end{array}$} & \multirow{2}{*}{$\begin{array}{l}\text { Class } \\
\text { C/classes } \\
\mathrm{A} \text { and } \mathrm{B}\end{array}$} & \multirow{2}{*}{$\begin{array}{c}\text { Classes C } \\
\text { and } \mathrm{G} / \\
\text { classes } \mathrm{A} \\
\text { and } \mathrm{B}\end{array}$} \\
\hline & $\begin{array}{l}\text { All class } \\
\text { A fatty } \\
\text { acids }\end{array}$ & $16: 0$ & $\begin{array}{l}\text { All class } \\
\text { C fatty } \\
\text { acids }\end{array}$ & $18: 3$ cis -9 & $\begin{array}{c}\mathrm{C}_{18} \\
\text { PUFA }\end{array}$ & & & & & & \\
\hline A. filiculoides & $33.3(\mathrm{a})^{b}$ & 31.2 (a) & 56.5 (a) & 27.4 (a) & 36.4 (a) & 3.1 (a) & 40.2 (a) & 44.2 (a) & 40.0 (a) & 1.7 (a) & 1.8 (a) \\
\hline A. microphylla & $36.7(a b)$ & 33.9 (a) & $57.1(\mathrm{a})$ & $25.0(\mathrm{a})$ & $35.4(\mathrm{a})$ & 0.6 (b) & 39.4 (a) & $47.1(\mathrm{a})$ & 40.9 (a) & 1.5 (a) & $1.6(\mathrm{~b})$ \\
\hline A. caroliniana & $37.4(\mathrm{bc})$ & $34.3(\mathrm{ab})$ & $54.4(\mathrm{ab})$ & 19.5 (b) & 32.2 (b) & 0.9 (b) & 36.4 (b) & 47.7 (a) & $38.4(\mathrm{ab})$ & $1.4(\mathrm{ab})$ & $1.5(\mathrm{~b})$ \\
\hline A. mexicana & $35.9(\mathrm{ab})$ & $32.3(\mathrm{a})$ & $53.1(\mathrm{~b})$ & $12.7(\mathrm{bc})$ & 24.9 (bc) & 0.9 (b) & $31.7(\mathrm{c})$ & $48.9(\mathrm{ab})$ & 32.5 (b) & $1.5(\mathrm{a})$ & $1.5(\mathrm{~b})$ \\
\hline A. rubra & $41.2(\mathrm{c})$ & $35.5(\mathrm{bc})$ & $51.2(\mathrm{~b})$ & $17.0(\mathrm{bc})$ & $25.8(\mathrm{bc})$ & $0.5(b)$ & $30.6(c)$ & $49.8(\mathrm{ab})$ & 35.2 (b) & $1.2(\mathrm{~b})$ & $1.2(\mathrm{c})$ \\
\hline A. nilotica & $42.2(\mathrm{c})$ & $36.8(\mathrm{bc})$ & $49.0(c)$ & $10.0(\mathrm{c})$ & $19.4(\mathrm{bc})$ & $0.9(b)$ & 27.1 (d) & 53.5 (b) & $28.3(\mathrm{c})$ & $1.1(b)$ & $1.2(\mathrm{c})$ \\
\hline A. pinnata ${ }^{c}$ & $42.9(\mathrm{c})$ & 39.8 (c) & $46.1(\mathrm{c})$ & $13.4(\mathrm{c})$ & $21.8(\mathrm{c})$ & 0.9 (b) & $27.6(\mathrm{~d})$ & $54.3(\mathrm{~b})$ & $29.1(\mathrm{c})$ & $1.1(\mathrm{~b})$ & $1.1(\mathrm{c})$ \\
\hline
\end{tabular}

${ }^{a}$ The values are the means for the fatty acid contents of the strains obtained from the hosts listed in Table 1 . The class A fatty acids are the saturated, even-carbon-number, straight-chain fatty acids; the class B fatty acids are the saturated, odd-carbon-number, straight-chain fatty acids; the class C fatty acids are the unsaturated, straight-chain fatty acids; and the class $\mathrm{G}$ fatty acids are the unsaturated, branched-chain fatty acids. PUFA, polyunsaturated fatty acid.

${ }^{b}$ In each column values that are not followed by the same letter are statistically different at least at the $0.05 \%$ level.

c Combined data for all symbiont strains obtained from the two subspecies of $A$. pinnata. 


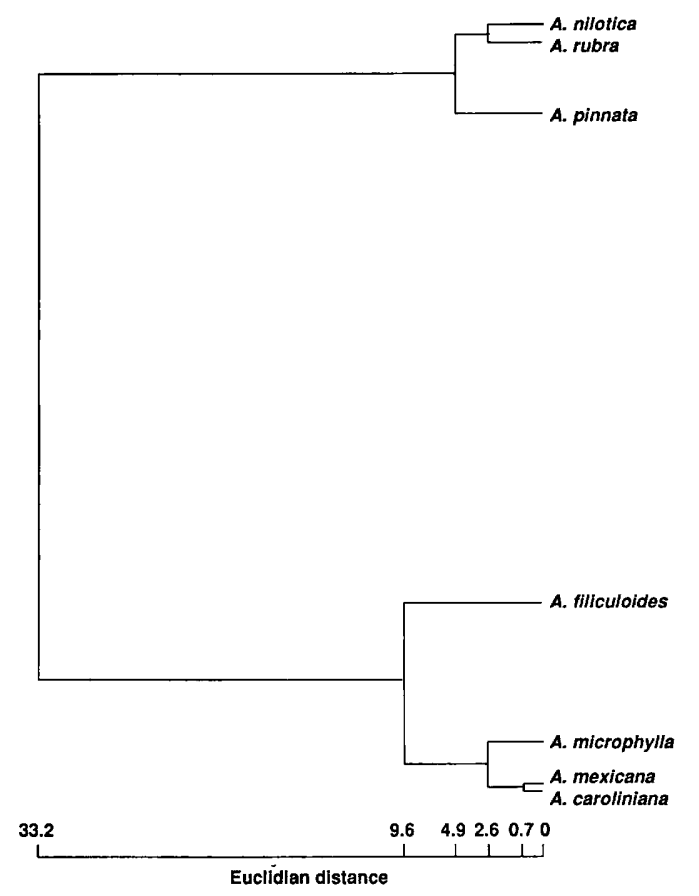

FIG. 1. Dendrogram based on the results of a cluster analysis of Euclidian distances between cyanobacteria obtained from seven Azolla host species. In this analysis we used average fatty acid values that were statistically different in symbionts of different host species (see Table 3). The horizontal distances are Euclidian distances between groups. The Azolla host species used were $A$. filiculoides, $A$. microphylla, $A$. caroliniana, $A$. mexicana, $A$. rubra, $A$. nilotica, and $A$. pinnata.

cyanobacterial symbionts isolated from members of the genus Azolla. These differences may be due to differences in the ecological niches in which the symbiotic cyanobacteria develop, since each Azolla species may be assumed to provide a distinct physiological, nutritional, and genetic environment. Morphologically, the Azolla cyanobacterial symbionts appear to be highly variable within certain parameters and also exhibit differences as a function of the host species (1).

The clustering of Azolla cyanobacterial symbionts which we observed based on the results of a fatty acid analysis correlates remarkably well with genetic clustering of selected tropical and temperate American Azolla species. Zimmerman et al. (25-27) reported that $A$. filiculoides had a distinct DNA fingerprint that could be distinguished from the fingerprint of a group composed of $A$. caroliniana, $A$. mexicana, and $A$. microphylla. In addition, $A$. rubra, was found to be distinct from the latter group. Similarly, the fatty acid composition of $A$. filiculoides symbionts was different from the fatty acid composition of symbionts of $A$. caroliniana, $A$. mexicana, and $A$. microphylla, all of which were separated from a secondary Azolla subgroup. As was found in the DNA analysis, $A$. rubra was a member of the second group (1). This correlation between cyanobacterial symbiont fatty acid composition and host genetics suggests that the Azolla cyanobacteria coevolved into distinct groups along with their hosts.

While it is difficult to detect and prove coevolution, there are other lines of evidence that strengthen the concept with regard to the Azolla cyanobacteria. Although there is no mention of symbiotic cyanobacteria in studies of the Azolla fossil record (12), the following characteristics suggest that the symbiotic relationship evolved at an early stage: (i) the obligate nature of the cyanobacterial partners in the symbiosis $(18,20)$; (ii) the synchronization of cyanobacterial and plant life cycles (18); and (iii) the fact that cyanobacteria caused induction and buildup of carbohydrates in plants utilized for bacterial growth (4). In addition, coevolution could have been driven by geographic isolation of the ferns since some members of the subgenus Azolla (A. caroliniana, A. mexicana, A. microphylla, and $A$. filiculoides) are found in the Americas and $A$. rubra occurs in Australia and New Zealand. The subgenus Rhizosperma contains $A$. nilotica (which occurs in Africa) and $A$. pinnata (which occurs in Africa and Asia).

Analyses of the cellular fatty acid profiles of members of the closely related but free-living cyanobacterial genera Anabaena and Nostoc revealed similarities with, but also significant differences from, the fatty acid profiles of the Azolla cyanobacterial symbionts (3). The Azolla cyanobacteria, therefore, may be a distinct taxon and may even be composed of different subspecies, each of which correlates with one of the dichotomous groups detected in the fatty acid analysis.

There is restriction fragment length polymorphism evidence that Azolla cyanobacterial symbionts arose from a common ancestor (10). Some morphological data, such as the occurrence of double heterocysts and synchronized cell expansion, support the hypothesis that these organisms had a common ancestor (1). Furthermore, division of the cyanobacteria into subgenera (whose members occur in members of Azolla subgenera Azolla and Rhizosperma) has been confirmed by morphological characteristics, including early hormogonium cell growth and differences in the large akinetes, and $\mathrm{G}+\mathrm{C}$ contents (1).

Regarding the evolution of the host species, Hills and Gopal (12) presented an evolutionary scheme in which the subgenus Azolla gave rise to the subgenus Rhizosperma. According to the available fossil record, the possible ancestor of the subgenus Rhizosperma is an unknown species (no fossil record) which lived during the Upper Cretaceous Period $\left(100 \times 10^{6}\right.$ to $80 \times$ $10^{6}$ years ago). Division into two subgenera, Azolla and Rhizosperma, may have started in the Mesozoic Era, with progressive changes in float number and glochidium (i.e., sexual organ) development continuing until relatively recent times (12). It is noteworthy that the cyanobacterial symbionts of $A$. rubra are considered more closely related to symbionts of species belonging to the subgenus Rhizosperma than to symbionts of species belonging to the subgenus Azolla. A. rubra is placed in the subgenus Azolla because of a general morphological resemblance to $A$. caroliniana, $A$. filiculoides, and $A$. microphylla. However, Mahabalé (14) found that $A$. rubra has characteristics of Azolla primaeva and Azolla prisca, which are extinct species which lived during the Eocene and Oligocene periods $\left(49 \times 10^{6}\right.$ to $30 \times 10^{6}$ years ago). A. primaeva and $A$. prisca are transitional species whose descriptions are based on reductions in the structure of the glochidia. Therefore, $A$. rubra may be a link between the subgenera Azolla and Rhizosperma or may belong to a new subgenus whose origin is different from that of the other subgenera.

\section{ACKNOWLEDGMENTS}

We acknowledge the contributions of Gerald Peters (Virginia Commonwealth University, Richmond), Charles Van Hove (Université Catholique de Louvain, Louvain-la-Neuve, Belgium), William Zimmerman (University of Michigan, Dearborn), Roxana Olvera (Mexico City, Mexico), William Rains (University of California, Davis), and Sandra Nierzwicki-Bauer (Rensselaer Polytechnic Institute, Troy, N.Y.), who provided some of the Azolla plants used in this study. We thank Thomas Hartman and Joseph Lech (Center for Advanced Food Technology, Rutgers-The State University of New Jersey, New Brunswick) and Larry G. Revear (U.S. Department of Agriculture) for technical assistance. We also thank Peter Anderson (Department of 
Biochemistry and Microbiology, Rutgers-The State University of New Jersey, New Brunswick) for word processing.

\section{REFERENCES}

1. Caudales, R. 1994. Characterization of the symbiotic cyanobacteria in Azolla: morphology, cellular fatty acids, DNA base composition and taxonomy. Ph.D. dissertation. Rutgers-The State University of New Jersey, New Brunswick.

2. Caudales, R., A. D. Antoine, and A. C. Vasconcelos. 1990. Morphology and life cycle of the nitrogen-fixing cyanobionts in the seven Azolla species, p. 540. In P. M. Gresshoff, L. Evans Roth, G. Stacey, and W. E. Newton (ed.), Nitrogen fixation: achievements and objectives. Proceedings of the 8th International Congress on Nitrogen Fixation. Chapman and Hall, New York.

3. Caudales, R., A. D. Antoine, and J. M. Wells. 1992. Cellular fatty acid composition of symbiotic cyanobacteria isolated from the aquatic fern Azolla. J. Gen. Microbiol. 138:1489-1494.

4. Caudales, R., G. Mohabir, J. M. Wells, and A. D. Antoine. 1993. Metabolic and osmoregulatory adjustments in Azolla-cyanobacteria symbiotic relationship, p. 255-260. In S. Sato, M. Ishida, and H. Ishikawa (ed.), Endocytobiology V. Tübingen University Press, Tübingen, Germany.

5. Caudales, R., and J. M. Wells. 1992. Differentiation of the free-living Anabaena and Nostoc cyanobacteria on the basis of fatty acid composition. Int. J. Syst. Bacteriol. 42:246-251.

6. Caudales, R., J. M. Wells, J. E. Butterfield, and A. D. Antoine. 1993. Hostrelated variability in fatty acid composition of symbiotic cyanobacteria isolated from the aquatic fern, Azolla, p. 261-268. In S. Sato, M. Ishida, and H. Ishikawa (ed.), Endocytobiology V. Tübingen University Press, Tübingen, Germany.

7. Duckett, J. G., R. Toth, and S. L. Soni. 1975 . An ultra-structural study of the Azolla-Anabaena azollae relationship. New Phytol. 75:111-118.

8. Fogg, G. E., W. D. P. Steward, P. Fay, and B. Walsby. 1973. The blue-green algae. Academic Press, New York.

9. Franche, C., and G. Cohen-Bazire. 1985. The structural nif genes of four symbiotic Anabaena azollae show higher conserved physical arrangement. Plant Sci. (Lucknow) 39:125-131.

10. Franche, C., and G. Cohen-Bazire. 1987. Evolutionary divergence in the nif $K, D$ and $H$ genes region among nine symbiotic Anabaena azollae and some free-living heterocystous cyanobacteria. Symbiosis 3:159-178.

11. Hill, D. J. 1975. The pattern of development of Anabaena in the AzollaAnabaena symbiosis. Planta (Berlin) 122:179-184.

12. Hills, L. V., and B. Gopal. 1967. Azolla primaeva and its phylogenetic significance. Can. J. Bot. 45:1179-1191.
13. Lumpkin, T. A., and D. L. Plucknett. 1982. Azolla as a green manure: use and management in crop production. Westview Press, Boulder, Colo.

14. Mahabalé, T. S. 1963 . Evolutionary tendencies in the genus Azolla. Indian Bot. Soc. Memorial 4:51-54.

15. Meeks, J. C., C. M. Joseph, and R. Haselkorn. 1988. Organization of nif genes in cyanobacteria in symbiotic association with Azolla and Anthoceros. Arch. Microbiol. 150:61-71.

16. Moore, A. W. 1969. Azolla: biology and agronomic significance. Bot. Rev. 35:17-34.

17. Perkins, S. K., G. A. Peters, T. A. Lumpkin, and H. E. Calvert. 1985. Scanning electron microscopy of perine architecture as a taxonomic tool in the genus Azolla Lamarck. Scanning Electron Microsc. 4:1719-1734.

18. Peters, G. A. 1991. Azolla and other plant-cyanobacteria symbiosis: aspects of form and function, p. 377-388. In M. Polsinelli, R. Materassi, and M. Vincenzini (ed.), Nitrogen fixation. Proceedings of the Fifth International Symposium on Nitrogen Fixation with Non-legumes. Kluwer Academic Publishers, Dordrecht, The Netherlands.

19. Peters, G. A., and B. C. Mayne. 1974. The Azolla-Anabaena relationship. I. Initial characterization of the association. Plant Physiol. 53:813-819.

20. Peters, G. A., and J. C. Meeks. 1989. The Azolla-Anabaena symbiosis: basic biology. Annu. Rev. Plant Physiol. 40:193-210.

21. Plazinski, J., C. Franche, C. C. Liu, T. Lin, W. Shaw, B. E. S. Gunning, and B. G. Rolfe. 1988. Taxonomic status of Anabaena azollae: an overview. Plant Soil 108:185-190.

22. Plazinski, J., G. Zheng, R. Tailor, L. Croft, B. G. Rolfe, and B. E. S. Gunning. 1990. DNA probes show genetic variation in cyanobacterial symbionts of the Azolla fern and closer relationship to free-living Nostoc strains than to free-living Anabaena strains. Appl. Environ. Microbiol. 56:12631270 .

23. Rai, A. N. (ed.). 1990. Cyanobacteria in symbiosis, p. 1-7. In CRC handbook of symbiotic cyanobacteria. CRC Press, Boca Raton, Fla.

24. Snedecor, G. W. 1966. Statistical methods, 5th ed. Iowa State University Press, Ames.

25. Zimmerman, W. J., T. A. Lumpkin, and I. Watanabe. 1989. Classification of Azolla spp., section Azolla. Euphytica 43:223-232.

26. Zimmerman, W. J., I. Watanabe, and T. A. Lumpkin. 1991. The AnabaenaAzolla symbiosis: diversity and relatedness of neotropical host taxa. Plant Soil 137:167-170.

27. Zimmerman, W. J., I. Watanabe, P. Ventura, P. Payawal, and T. A. Lumpkin. 1991. Aspects of the genetic and botanical status of neotropical Azolla species. New Phytol. 119:561-566. 